\title{
Primary Seminal Vesicle Adenocarcinoma Presenting as Acute Urinary Retention and Hematuria: A Case Report
}

\author{
Khaireddine Bouassida ${ }^{\mathrm{a}, \mathrm{c}}$, Wissem Hmida ${ }^{\mathrm{a}}$, Sarra Mestiri ${ }^{\mathrm{b}}$, Ghassen Tlili ${ }^{\mathrm{a}}$, \\ Moncef Mokni ${ }^{\mathrm{b}}$, Ali Tahar Mosbah ${ }^{\mathrm{a}}$
}

\begin{abstract}
Primary tumors of the seminal vesicle are extremely rare lesions, and adenocarcinoma is the most common histology found. We report the case of a 54-year-old patient who has consulted with a bladder outlet obstruction and two episodes of initial hematuria for 3 months. Digital rectal examination revealed a hard, palpable mass in the prostate wall. The prostate-specific antigen (PSA), the serum carcinoembryonic antigen and the cancer antigen 125 (CA125) were normal. On imaging, a prostatic abscess, granulomatous prostatitis or a prostatic cancer were highly suspected at first. But the patient was afebrile and PSA was normal. Transrectal ultrasound-guided biopsies of the mass were performed. The pathological examination showed a poorly differentiated adenocarcinoma. Immunohistological analysis was strongly suggestive of adenocarcinoma of the seminal vesicle. At surgical exploration, it was impossible to make cystoprostatovesiculectomy because the tumor was locally advanced. We decided to make hormonal blockage by antiandrogen and five sessions of radiotherapy. Unfortunately, the patient died 5 months later. Adenocarcinoma of the seminal vesicles is a rare diagnosis. We must think in this diagnosis when relatively young patient is presented with dysuria, hematuria or hemospermia and when we found hard palpable mass on digital rectal examination associated to normal PSA level. On histology, specific immunohistochemical markers would be helpful in this differential diagnosis. Actually, surgical excision is the mainstay of the treatment. Radiotherapy and androgen deprivation therapy can be an alternative in locally advanced and metastatic carcinomas.
\end{abstract}

Keywords: Adenocarcinoma; Differential diagnosis; Seminal vesicle; MRI; Urogenital neoplasm

Manuscript accepted for publication February 18, 2015

aDepartment of Urology, Hospital of Sahloul, Sousse, Tunisia bepartment of Pathology, Hospital of Farhat Hached, Sousse, Tunisia ${ }^{\mathrm{c} C}$ Corresponding Author: Khaireddine Bouassida, Department of Urology, Hospital of Sahloul, Rue de Tunis, Ksibet Sousse 4041, Tunisia.

Email: khayri14@hotmail.com

doi: http://dx.doi.org/10.14740/wjnu204e

\section{Introduction}

Primary tumors of the seminal vesicle are extremely rare lesions, adenocarcinoma is the most common histology found, and less than 100 cases were reported in the literature [1]. Symptoms are non-specific such as obstructive uropathy or hematuria and sometimes hemospermia. On radiology, it is difficult to distinguish primary carcinoma of the seminal vesicle from a local invasion from the prostate cancer. Specific immunohistochemical markers would be helpful in this differential diagnosis. Because it is often detected late, adenocarcinoma of the seminal vesicle has a poor prognosis. Currently there is no standard treatment especially for locally advanced and metastatic diseases; however, surgery is recommended for localized disease and radiotherapy can be associated when surgical margins are positive. In this case, primary seminal vesicle adenocarcinoma will be discussed with a review of current literature.

\section{Case Report}

A 54-year-old patient has consulted with a bladder outlet obstruction and two episodes of initial hematuria for 3 months.

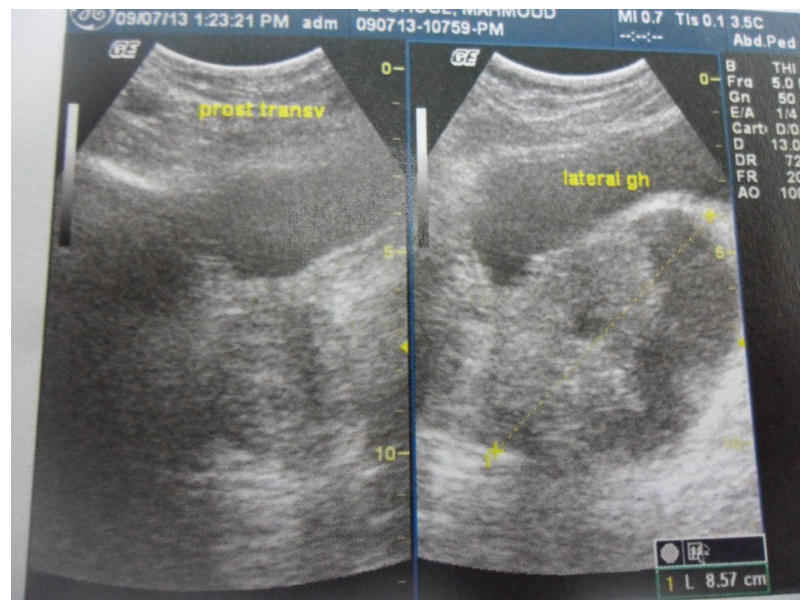

Figure 1. Pelvic ultrasound. An $8.5 \mathrm{~cm}$ heterogeneous cystic and solid mass in the left periprostatic region. 


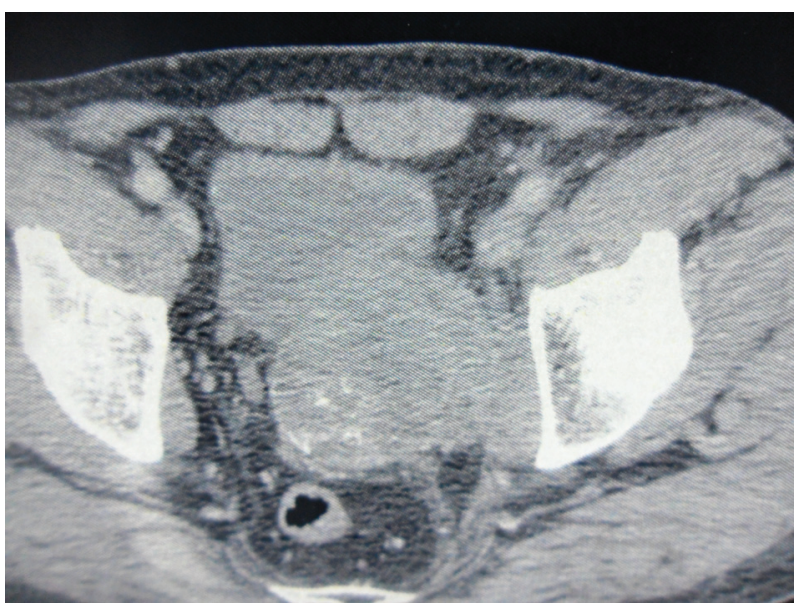

Figure 2. Pelvic computed tomography. An $8.5 \times 6 \mathrm{~cm}$ solid and heterogeneous mass in the left superior aspect of the prostate with cystic area and peripheral calcifications.

The patient has no medical or surgical past history. The patient was afebrile, digital rectal examination revealed a hard, palpable mass, and no other abnormality was noticed in physical examination.

The prostate-specific antigen (PSA) was normal $(2.7 \mathrm{ng} /$ $\mathrm{mL}$ ), and the serum carcinoembryonic antigen (CEA) and the cancer antigen 125 (CA125) were also normal respectively 4.7 $\mathrm{ng} / \mathrm{mL}$ and $23.4 \mathrm{U} / \mathrm{mL}$.

Pelvic ultrasound showed an $8.5 \mathrm{~cm}$ heterogeneous cystic and solid mass under the bladder and in the left periprostatic region (Fig. 1). A prostatic abscess or granulomatous prostatitis was highly suspected at first.

Computed tomography showed an $8.5 \times 6 \mathrm{~cm}$ solid and heterogeneous mass on the left superior aspect of the prostate with cystic area and peripheral calcifications (Fig. 2).

A pelvic magnetic resonance imaging (MRI) was performed to further characterize this mass.

It demonstrated a $10 \times 9 \times 12 \mathrm{~cm}$ solid, poorly defined and heterogeneous mass on the left seminal vesicle wall. The

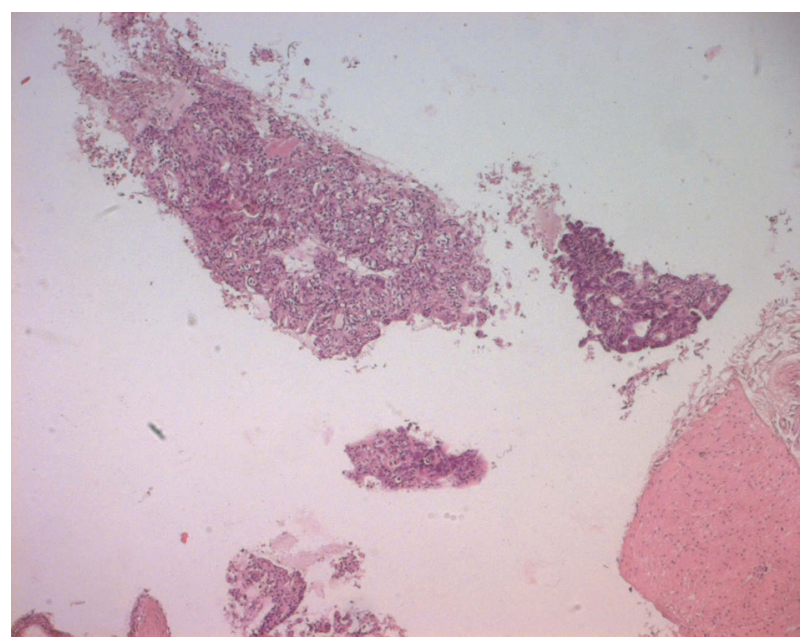

Figure 4. Echo-guided transrectal biopsy (low magnification). Pathological tissue with papillary and pseudo glandular structure, normal prostatic tissue, normal seminal vesicle tissue.

mass was hypo-signal in $\mathrm{T} 1$ sequences and hyper-signal in $\mathrm{T} 2$ sequences compared to the muscle and has an important local invasion. The mass invades the posterior and lateral bladder wall, the prostate base, the internal obdurate and serratus muscle and the hip bone, and the external and internal iliac lymph nodes were also invaded (Fig. 3).

Transrectal ultrasound-guided biopsies of the mass were performed.

The pathological examination showed large and medium tumor cells with rounded nucleus and transparent cytoplasm, papillary and glandular structures were observed, and a normal seminal vesicle and prostate tissue were also found. This shows a poorly differentiated adenocarcinoma, but its origin was not yet clear (Fig. 4, 5).

Immunohistochemical evaluation demonstrated that cytokeratin (CK) 7 was positive while CEA, CK20, PSA, CA125 and chromogranin were negative (Fig. 6).

Immunohistological analysis is strongly suggestive of ad-
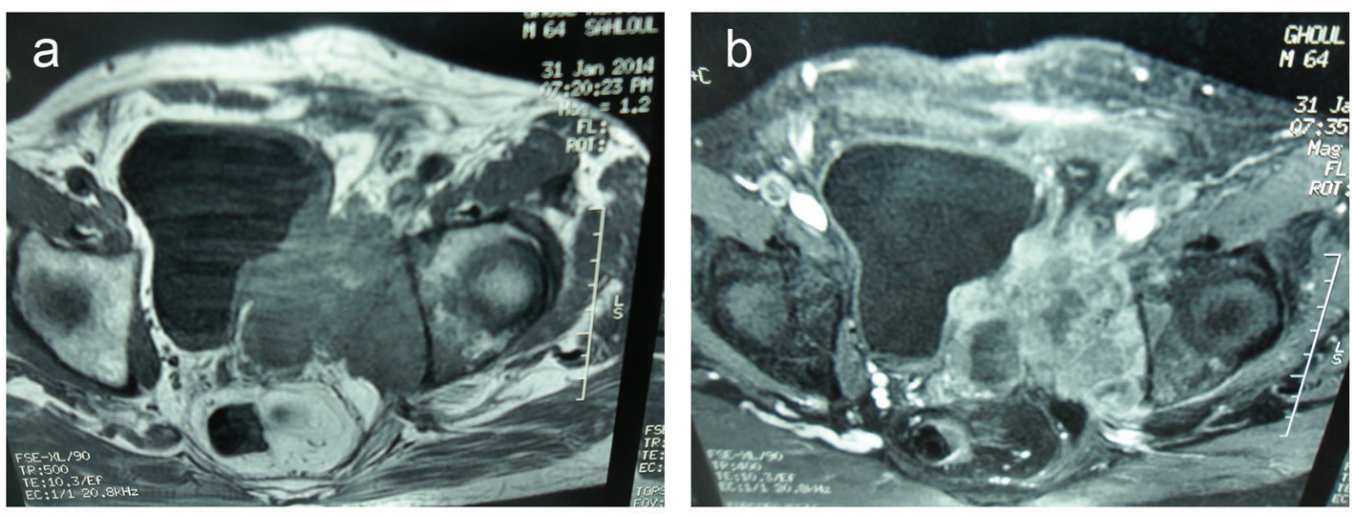

Figure 3. (a) Transverse T2-weighted MR images and transverse T1-weighted MR images (b). A $10 \times 9 \times 12 \mathrm{~cm}$ solid and heterogeneous mass in the left seminal vesicle wall. The mass was hypointense in T1-weighted and hyperintense in T2-weighted MR images compared to the muscle. The mass invades the posterolateral bladder wall, the prostate base, the internal obturator and serratus muscles and the hip bone. 


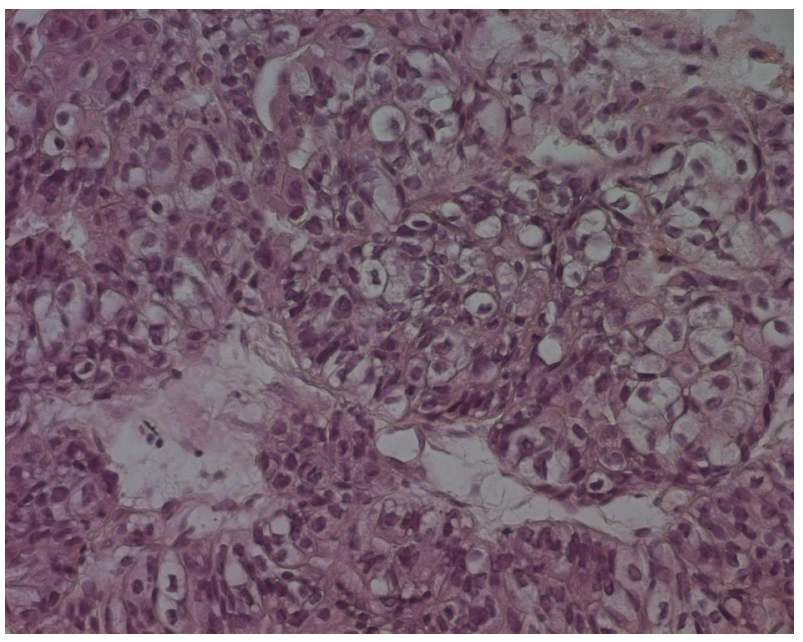

Figure 5. Echo-guided transrectal biopsy (high magnification). Large and medium tumor cells with rounded nucleus and transparent cytoplasm, papillary and glandular structures can be also found.

enocarcinoma of the seminal vesicle.

A rectoscopy and a cystoscopy were done and revealed no tumors in the rectum and in the bladder respectively.

According to those results, the patient was diagnosed with a seminal vesicle locally advanced adenocarcinoma.

At surgical exploration, it was impossible to make cystoprostato-vesiculectomy because the tumor was locally advanced.

We decided to make hormonal blockage by antiandrogen and five sessions of radiotherapy. Unfortunately, the patient died 5 months later.

\section{Discussion}

Malignant lesions of the seminal vesicles are most often the result of secondary invasion from other tumors, most commonly prostate, rectum and bladder cancer. Primary adenocarcinoma of the seminal vesicle is an extremely rare neoplasm with fewer than 100 reported cases in the literature [1].

In 1956, Dalgaard and Giertson [2] established the following criteria for a diagnosis of SVC: 1) The tumor should be a microscopically verified carcinoma, localized exclusively or mainly in the seminal vesicle. 2) The presence of other simultaneous primary carcinomas should be excluded. 3) The

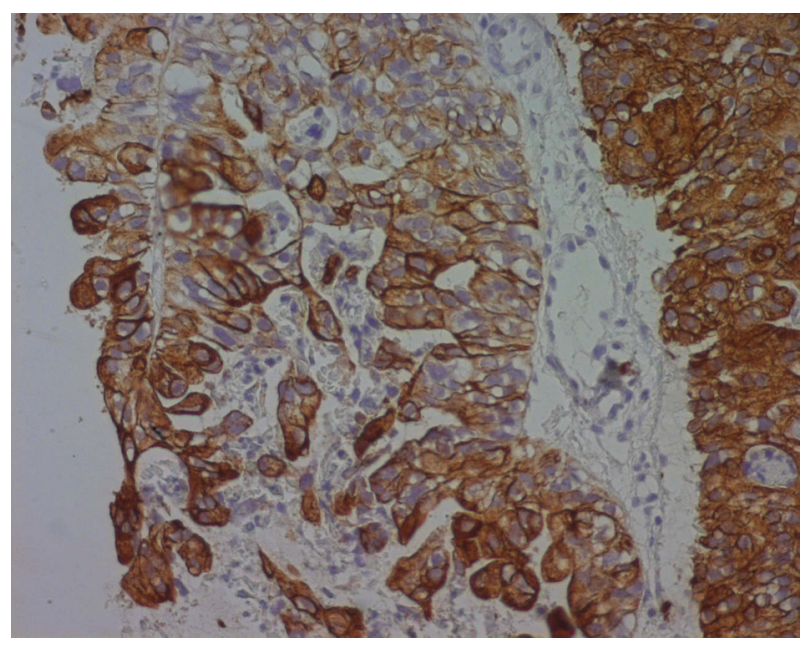

Figure 6. Cytokeratin 7 immunoreactivity in a poorly differentiated seminal vesicle adenocarcinoma. The immunoreactivity appears as a strong brownish cytoplasmic staining in neoplastic cells.

tumor should preferably resemble the architecture of the nonneoplastic seminal vesicle.

Although seminal vesicle adenocarcinoma can be observed in males at any age, the mean age at the time of diagnosis is 62 years (ranging from 13 to 90 years) [3]. Symptoms are generally non-specific and often reflect an advanced disease with infiltration of adjacent organs obscuring the vesicular origin of the tumor. Most common presentations include bladder outlet obstruction, hematuria, hematospermia, dysuria and painful sensation in the pelvis and perineum. Digital rectal examination and cystoscopy may be useful; however, these are normal in up to $20-30 \%$ of cases $[1,3]$.

Ultrasonography, CT scan, and MRI may be helpful in localizing the tumor and to evaluate its local extension. It can also help detect a primary lesion or any associated congenital genitourinary malformations such as ectopic ureters or renal agenesis. This malformation can be associated with seminal vesicle cysts, dysplasia and tumors.

On imaging, seminal vesicle tumors may appear as a retrovesical mass with or without prostatic invasion and ureteral obstruction. It may also appear as an infiltrating lesion in the seminal vesicle with enhancement similar to that found in advanced prostate cancer. Vesical seminal tumors are usually solid or cystic masses poorly circumscribed that may be misinterpreted as an abscess or hemorrhage [1,4].

Table 1. Immunohistological Findings in Carcinomas of Seminal Adenocarcinoma and the Most Common Carcinomas in Other Pelvic Organs

\begin{tabular}{llllll}
\hline & $\begin{array}{l}\text { Seminal vesical } \\
\text { adenocarcinoma }\end{array}$ & $\begin{array}{l}\text { Prostate } \\
\text { carcinoma }\end{array}$ & $\begin{array}{l}\text { Bladder } \\
\text { cancer }\end{array}$ & $\begin{array}{l}\text { Rectum } \\
\text { cancer }\end{array}$ & $\begin{array}{l}\text { Adenocarcinoma of the } \\
\text { Mullerian duct }\end{array}$ \\
\hline CA125 & + & - & - & - & \pm \\
Cytokeratin 7 & + & \pm & \pm & - & + \\
PSA & - & + & - & - & - \\
Cytokeratin 20 & - & \pm & \pm & + & - \\
CEA & \pm & - & - & + & \pm \\
\hline
\end{tabular}


Specific immunohistochemical markers would be helpful in this difficult diagnosis because the seminal vesicle may be invaded by tumors of adjacent organs, particularly those of the prostatic adenocarcinoma. In fact, the diagnostic criteria for seminal vesicle adenocarcinoma were modified by Benson et al [5] and the seminal vesicle should be verified macroscopically and microscopically to confirm this diagnosis. Moreover, to confirm that the tumor is completely or essentially localized to the seminal vesicles, PSA, prostate-specific acid phosphatase (PAP), and CEA should be negative. However, increased serum CEA levels can also be observed in rare cases of seminal vesicle adenocarcinoma [6]. Absence of staining of PSA and PAP helps to distinguish seminal vesical adenocarcinoma from prostatic adenocarcinoma [7]. The other differential diagnoses are tumors of other organs adjacent to the seminal vesicles, specifically bladder transitional cell carcinoma (TCC) and rectal adenocarcinoma. Immunohistochemical stains using monoclonal antibodies directed against $\mathrm{CK}$ subsets 7 and 20 have demonstrated specificity for bladder TCC and rectal adenocarcinoma [3], as bladder TCC is CK7 and CK20 positive, whereas rectal adenocarcinoma demonstrates only CK20 immunoreactivity. However, only CK7 and CA125 are positive in cases of seminal vesical adenocarcinoma and CK20 is always negative $[7,8]$ (Table 1 ), in our case only CK7 was positive and all other biomarkers were negative.

Because of the rarity of this tumor, there are not much data regarding management protocols and guidelines for the treatment and each case of seminal vesicle adenocarcinoma is treated individually.

Treatment strategies have focused on surgical excision of the tumor. Extensive pelvic lymph node dissection is also recommended. Only tumors without prostatic involvement might be treated with local excision or with vesiculectomy only, this clinical scenario rarely occurs and ejaculatory canals are frequently invaded; therefore, prostatectomy should also be performed. Radiotherapy can be applied as another treatment modality in the presence of postoperative positive surgical borders or residual tumor $[9,6]$.

Androgen deprivation therapy may also be beneficial in the adjuvant or palliative settings, and in fact several small reports suggest that its use may increase disease-free survival [4, 10]. Radiotherapy has been used with limited success, mostly in combination with surgical or androgen deprivation therapy. There are a few reports of multiagent chemotherapeutic regimens attempted for the treatment of disseminated disease. However, the results are disappointing [1]. Chemotherapy can be used as an adjuvant treatment or as a neoadjuvant treatment before surgery and in metastatic adenocarcinomas of the seminal vesicle. The most active chemotherapeutic agents are vincristine, actinomycin D, cyclophosphamide and doxorubicin. Ifosfamide and etoposide may be added. The optimal combination of chemotherapeutic drugs is still debated and should be individualized [2].

In our case, the patient received androgen deprivation therapy and radiotherapy because surgical excision was impossible but the result was disappointing and the patient died 5 months later.

The prognosis of seminal vesical adenocarcinoma is often poor due to delayed diagnosis and about $94 \%$ of the patients die in less than 3 years [6].

\section{Conclusion}

Adenocarcinoma of the seminal vesicles is a rare diagnosis. The lack of early or specific clinical presentation leading to late identification of these tumors contributes to their poor prognosis. We must think in this diagnosis when relatively young patient is presented with dysuria, hematuria or hemospermia and when we found hard palpable mass on digital rectal examination associated to normal PSA level.

On histology, specific immunohistochemical markers would be helpful in this differential diagnosis. Actually, surgical excision of the seminal vesicle or radical prostatectomy is the mainstay of the treatment. Radiotherapy and androgen deprivation therapy can be an alternative in locally advanced and metastatic carcinomas.

\section{Acknowledgement}

We thank Dalel Mokni for writing assistance.

\section{Competing Interests}

The authors declare that they have no competing interests.

\section{Abbreviations}

PSA: prostate-specific antigen; CEA: carcinoembryonic antigen; CA125: cancer antigen 125; MRI: magnetic resonance imaging; TCC: transitional cell carcinoma; CK: cytokeratin

\section{References}

1. Lote H, Mannion E, Cook T, Cairns T, Savage P. Adenocarcinoma of the seminal vesicles complicated by antineutrophil cytoplasmic antibody vasculitis: a case report and review of the literature. J Med Case Rep. 2013;7:59.

2. Ramamurthy R, Periasamy S, Mettupalayam V. Primary malignancy of seminal vesicle: A rare entity. Indian J Urol. 2011;27(1):137-139.

3. Tarjan M, Ottlecz I, Tot T. Primary adenocarcinoma of the seminal vesicle. Indian J Urol. 2009;25(1):143-145.

4. Navallas M, Vargas HA, Akin O, Pandit-Taskar N, Fine SW, Eastham JA, Hricak H. Primary seminal vesicle adenocarcinoma. Clin Imaging. 2011;35(6):480-482.

5. Benson RC, Jr., Clark WR, Farrow GM. Carcinoma of the seminal vesicle. J Urol. 1984;132(3):483-485.

6. Eken A, Izol V, Aridogan IA, Erdogan S, Acikalin A, Tansug Z. An unusual cause of hematospermia: Primary adenocarcinoma of the seminal vesicle. Can Urol Assoc J. 2012;6(6):E259-262.

7. Ormsby AH, Haskell R, Ruthven SE, Mylne GE. Bi- 
lateral primary seminal vesicle carcinoma. Pathology. 1996;28(2):196-200.

8. Ormsby AH, Haskell R, Jones D, Goldblum JR. Primary seminal vesicle carcinoma: an immunohistochemical analysis of four cases. Mod Pathol. 2000;13(1):46-51.

9. Favaretto RL, Ercolani MC, Sanchez-Salas R, Vali- dire P, Barret E, Cathelineau X. [Management of primary carcinoma of the seminal vesicle]. Actas Urol Esp. 2012;36(1):67-68.

10. Gohji K, Kamidono S, Okada S. Primary adenocarcinoma of the seminal vesicle. Br J Urol. 1993;72(4):514515 . 